\title{
Hallazgos principales de la investigación "Balance crítico de las Pruebas Nacionales de Bachillerato en Educación Media como instrumento de certificación del conocimiento del estudiantado"
}

\section{"Critical Balance of Baccalaureate National Tests in High School as a Student' Knowledge Certification Tool" Core Findings}

\section{Principais resultados da pesquisa "Balance Crítico dos Testes de Bacharelado Nacional no Ensino Médio como instrumento para certificar o conhecimento dos alunos"}

Centro de Investigación y Docencia en Educación

Heredia, Costa Rica

francisco.gonzalez.alvarado@una.ac.cr

D http://orcid.org/0000-0001-8561-0411

Ignacio Acosta-Ballestero

Universidad Nacional

Centro de Investigación y Docencia en Educación

Heredia, Costa Rica

iacostaballestero86@gmail.com

https://orcid.org/0000-0002-3759-2826

Cindy Vanessa Artavia-Aguilar

Universidad Nacional

Centro de Investigación y Docencia en Educación

Heredia, Costa Rica

cindy.artavia.aguilar@una.cr

iD http://orcid.org/0000-0002-2179-6808

Guillermo Calderón-Ramírez

Universidad Nacional

Centro de Investigación y Docencia en Educación

Heredia, Costa Rica

gcalderon@una.cr

http://orcid.org/0000-0003-0830-3164

Pablo Chaverri-Chaves

Universidad Nacional

Centro de Investigación y Docencia en Educación

Heredia, Costa Rica

pablochaverri@yahoo.com

http://orcid.org/0000-0002-2639-4242

Francisco González-Alvarado, Ignacio Acosta-Ballestero, Cindy Vanessa Artavia-Aguilar, Guillermo Calderón-Ramírez, Pablo Chaverri-Chaves, Eddy Cruz-Arroyo, Andrea Ramírez-González, Magaly Rodríguez-Pineda y José Solano-Alpizar 
doi: http://doi.org/10.15359/ree.24-1.13

URL: http://www.una.ac.cr/educare

CORREO: educare@una.cr

Eddy Cruz-Arroyo

Universidad Nacional

Centro de Investigación y Docencia en Educación

Heredia, Costa Rica

ecruz@una.cr

http://orcid.org/0000-0003-1741-1677

Andrea Ramírez-González

Universidad Nacional

Centro de Investigación y Docencia en Educación

Heredia, Costa Rica

andrea.ramirez.gonzalez@una.cr

http://orcid.org/0000-0002-9497-0867

Magaly Rodríguez-Pineda

Universidad Nacional

Centro de Investigación y Docencia en Educación

Heredia, Costa Rica

magaly.rodriguez.pineda@una.cr

https://orcid.org/0000-0002-3990-2760

José Solano-Alpízar

Universidad Nacional

Centro de Investigación y Docencia en Educación

Heredia, Costa Rica

josesolanoalpizar@gmail.com

http://orcid.org/0000-0003-3819-8158

Recibido • Received • Recebido: 11 / 06 / 2019

Corregido • Revised • Revisado: 04 / 11 / 2019

Aceptado • Accepted • Aprovado: $30 / 11 / 2019$

Resumen: Este artículo es producto del quehacer investigativo del programa "Perfiles, dinámicas y desafíos de la educación costarricense", del Centro de Investigación y Docencia en Educación de la Universidad Nacional. Expone los principales resultados de un trabajo investigativo de carácter diagnóstico, con diferentes niveles de análisis: exploratorio, descriptivo y correlacional, que utilizó una estrategia mixta de triangulación concurrente, vinculando métodos cuantitativos y cualitativos. Participaron como informantes: exjerarcas del Ministerio de Educación Pública (MEP); directores y directoras regionales de educación; personal directivo de colegios públicos, privados, subvencionados, urbanos y rurales; especialistas en evaluación; representantes gremiales; docentes y estudiantes. Se realizaron entrevistas semiestructuradas y encuestas, se utilizaron los datos estadísticos del MEP sobre el rendimiento estudiantil en las pruebas de bachillerato, registros de carácter sociodemográfico y socioeducativo e índices sociales asociados a la población examinada. El análisis de los datos refleja algunos aspectos trascendentales en materia de equidad, inclusión educativa y condiciones de desarrollo social, para tomar en consideración en la interpretación de los resultados de las Pruebas Nacionales de Bachillerato en la Educación Media en Costa Rica.

Palabras claves: Educación media; evaluaciones nacionales; pruebas estandarizadas; educación inclusiva; evaluación de altas consecuencias. 


\begin{abstract}
This article is the product of the investigative activity of the Program Profiles, Dynamics and Challenges of Costa Rican Education, from the Center of Research and Teaching in Education (CIDE) from the National University of Costa Rica (UNA). This paper is a diagnostic work, with different levels of analysis: exploratory, descriptive, and correlational, which utilized a mixed method strategy of concurrent triangulation, incorporating quantitative and qualitative methods. Diverse informants participated; among them, there were former ministries of education, regional directors of the Ministry of Education (MEP), directors from public, private, subsidized, urban and rural secondary education centers, evaluation specialists, union representatives, professors, and students. Semistructured interviews and structured questionnaires were applied. Also, databases from MEP on student's performance in bachelor tests, sociodemographic and socioeducative data, and social indexes associated with the examined population were used. The analysis of data reflects some transcendental aspects in matters of educative equity, inclusion, and conditions of social development to consider for the interpretation of the results of the national bachelor test in the country.
\end{abstract}

Keywords: Secondary education; standardized tests; inclusive education; high consequences evaluation.

Resumo: Este artigo é produto do trabalho investigativo do Programa Perfis, Dinâmicas e Desafios da Educação Costarriquense, do Centro de Investigação e Docência em Educação da Universidade Nacional, em seu desenvolvimento apresenta-se um resumo geral da investigação. Este trabalho tem como objetivo contribuir, a partir de seus principais achados, com a discussão nacional em matéria de política educativa. É um estudo de caráter diagnóstico, com diferentes níveis de análises: exploratório, descritivo e correlação, utilizou-se a estratégia mista de triangulação concorrente fazendo uso de métodos quantitativos e qualitativos. Participaram Ex-ministros do MEP, diretores regionais de educação, diretores de colégios públicos, privados, subvencionados, urbanos e rurais, especialistas em avaliação, representantes gremial de educação, docentes e estudantes. Realizaramse entrevistas semiestruturadas, questionário, utilizaram-se os dados estatísticos do rendimento da população estudantil, registros de caráter sociodemográficos, socioeducativos e índices sociais unidos à população examinada. A análise dos dados reflete-nos algumas discussões de importância em matéria de equidade, inclusão educativa e condições de desenvolvimento social a tomar em consideração para a interpretação dos resultados das PNB-EM no país.

Palavras chaves: Ensino médio; avaliação; testes padronizados; educação inclusiva; avaliação de altas consequências.

\title{
Introducción
}

El presente artículo discute los principales hallazgos de la investigación Balance crítico de objetivos, modelo evaluativo, resultados y utilidad de la información de las Pruebas Nacionales de Bachillerato en Educación Media (PNB-EM) como instrumento de certificación del conocimiento de estudiantes, desarrollada en el proyecto de investigación Perfiles, dinámicas y desafíos de la educación costarricense, adscrito al Instituto de Estudios Interdisciplinarios de la Niñez y la Adolescencia (INEINA) del Centro de Investigación y Docencia en Educación (CIDE) de la Universidad Nacional, durante el 2017 y el 2018.Esta información está asociada con la realización 
doi: http://doi.org/10.15359/ree.24-1.13

URL: http://www.una.ac.cr/educare

CORREO: educare@una.cr

de PNB-EM, reestablecidas en el país desde el año 1988 y que, para la aplicación del 2019, luego de 30 años de realización ininterrumpida, serán modificadas sustantivamente y sustituidas por las Pruebas Nacionales para el Fortalecimiento de Aprendizajes para la Renovación de Oportunidades (FARO), por acuerdo del Consejo Superior de Educación (CSE) CSE-SG-143-2019 (CSE, 2018). Esta decisión, tal y como recoge un acuerdo previo de este órgano de mayor jerarquía en educación a nivel nacional, encontró en la investigación que sustenta la presente publicación: “...resultados elocuentes y la información sumamente importante y definitoria..." (CSE, 2018, p. 1).

La investigación permitió arribar y documentar importantes conclusiones respecto a cuatro objetivos específicos de las PNB-EM: 1) examinar los objetivos orientadores de las PNBEM y la percepción de sujetos clave sobre estos mismos; 2) caracterizar el modelo evaluativo que sustentó su elaboración y ejecución; 3) establecer relaciones entre los resultados individuales y colectivos de la población estudiantil que realizó las PNB-EM y el índice de desarrollo social nacional (IDS-2017) del distrito de su procedencia, a partir de la definición de perfiles exploratorios relacionados con características sociodemográficas, socioeducativas y de rendimiento, para lo cual se seleccionó la convocatoria ordinaria de la modalidad académica y técnica del 2016, en vista de que un análisis preliminar evidenció una recurrencia, al menos en los últimos 5 años, de los resultados obtenidos, y, finalmente, 4) valorar el uso de la información derivada de los resultados por parte de los sectores involucrados en el proceso educativo; elementos todos que facilitaron concretar un análisis desde una perspectiva más integral.

Estudios previos tales como: "Estudio correlacional entre las Pruebas de Bachillerato en la Educación Media y el rendimiento en Educación Diversificada" (Moreira, 2002); Evaluación externa de las Pruebas Nacionales de bachillerato de la Educación Media (Esquivel et al., 2006); así como también, otras realizadas por el Estado de la Educación Costarricense (Programa Estado de la Nación, 2005) y los informes técnicos producidos anualmente por la Dirección de Gestión y Evaluación de la Calidad (DGEC) del MEP, identificaron importantes brechas en los resultados obtenidos por la población estudiantil que las realizó en diferentes cohortes, especialmente documentan diferencias sustantivas en cuanto al financiamiento de las instituciones: pública, privada y subvencionada. No obstante, las asociaciones entre los resultados de las PNB-EM y las condiciones de desarrollo social del lugar de residencia de la población estudiantil examinada no habían sido estudiadas con suficiente profundidad, limitación que la investigación en cuestión abordó con especial énfasis, al aportar argumentaciones sustentadas en análisis estadísticos y entrevistas en profundidad a personas involucradas directas, que tal y como se expondrán más adelante, permiten aseverar que las PNB-EM constituyeron evaluaciones de altas consecuencias y que revelan desigualdades educativas, entre la población estudiantil. Estos hallazgos han sido publicados en artículos científicos: Artavia-Aguilar et al. (2019), Chaverri, Cruz y González (2018). En consecuencia, el artículo se divide en 4 apartados, además de la introducción: 1) referentes conceptuales; 2) presupuestos metodológicos; 3) discusión de los principales hallazgos (presentados de acuerdo con cada uno de los objetivos) y 4) las conclusiones generales.

4

Francisco González-Alvarado, Ignacio Acosta-Ballestero, Cindy Vanessa Artavia-Aguilar, Guillermo Calderón-Ramírez, Pablo Chaverri-Chaves, Eddy Cruz-Arroyo, Andrea Ramirez-González, Magaly Rodríguez-Pineday José Solano-Alpizar

Los artículos de la Revista Electrónica Educare del Centro de Investigación y Docencia en Educación de la Universidad Nacional, Costa Rica, se comparten bajo términos de la Licencia Creative Commons: Reconocimiento, No Comercial, Sin Obra Derivada 3.0 Costa Rica. Las autorizaciones adicionales a las aquí delimitadas se pueden obtener en el correo: educare@una.cr 


\section{Referentes conceptuales}

Las PNB-EM constituyen evaluaciones estandarizadas de altas consecuencias sociales, cuyos resultados, en términos del rendimiento obtenido por la población estudiantil que las realiza, están asociados con diferentes factores, entre ellos; el tipo de oferta educativa y las condiciones de desarrollo social del lugar de residencia. De igual forma, las razones que justificaron su puesta en marcha están vinculadas con las políticas educativas nacionales adoptadas en un periodo histórico particular. En consecuencia, es imprescindible discutir sobre estos elementos, con el objeto de poder construir el andamiaje teórico-conceptual referencial de los resultados más adelante presentados.

En primer instancia, es importante considerar que las políticas educativas constituyen herramientas específicas dentro de las políticas públicas nacionales, cuyo objetivo es establecer medidas de carácter general, sobre la educación en un sentido amplio y sistemático, las cuales responden a determinados condicionamientos históricos, políticos, económicos y sociales. En este sentido, el MEP cita, en la Política Educativa que la persona es el centro del proceso educativo y sujeto transformador de la sociedad, en un sentido amplio; que la política educativa tiene por propósito "sistematizar [una] nueva visión en un cuerpo de política que la integre y le confiera coherencia, de forma que guíe el desarrollo presente y futuro de la educación nacional" (MEP, 2017b, p. 1). De ahí que la iniciativa de reinstaurar las PNB-EM respondiera a determinadas políticas educativas, que para las décadas de los 80 y 90 estuvieron influenciadas por las concepciones de calidad de la educación, diseminadas por organismos internacionales de la educación principalmente vinculados con la Organización de las Naciones Unidas para la Educación, la Ciencia y la Cultura (UNESCO) y cuyas expresiones en el ámbito costarricense se materializaron fundamentalmente en La política educativa Hacia el Siglo XXI, puesta en ejecución desde 1994 y con vigencia hasta el 2017.

Con el amparo de la política educativa citada, a la evaluación de tipo estandarizada propia de los modelos referidos a normas "se le atribuyen fortalezas como la interpretación objetiva de los resultados, la comparación del individuo con el resto del grupo y entre grupos, así como la utilidad para identificar o seleccionar individuos (por ejemplo: para certificación, admisión, becas, aprovechamiento escolar, entre otros propósitos)" (MEP, 2017a, p. 16). Este se constituyó en el tipo de medición que sustentó las PNB-EM y que por los resultados obtenidos a lo largo de 30 años de aplicación pueden catalogarse como evaluaciones de altas consecuencias. SánchezMendiola y Delgado-Maldonado (2017, p. 55), respecto a este tipo de evaluaciones, plantean:

La definición de EAI o de altas consecuencias («high-stakes testing» en inglés) de acuerdo con el Instituto Nacional para la Evaluación de la Educación en México: ... «indica cuando los resultados del instrumento tienen consecuencias importantes para las personas o las instituciones; por ejemplo, en los procesos de admisión o certificación» (INEE, 2014), y de 
doi: http://doi.org/10.15359/ree.24-1.13

URL: http://www.una.ac.cr/educare

CORREO: educare@una.cr

acuerdo a la edición 2014 de los Standards: «pruebas o exámenes cuyos resultados tienen consecuencias importantes y directas para los individuos, programas o instituciones involucrados en el examen» (American Educational Research Association, American Psychological Association and National Council on Measurement in Education y Joint Committee on Standards for Educational and Psychological Testing (2014).

El empleo de este tipo de pruebas no está libre de controversia y críticas, además, este argumento otorga un peso importante a los factores asociados con el proceso educativo (características curriculares de la oferta educativa) y al contexto socioeconómico y cultural. Respecto al primero, se reconoce que el sistema educativo costarricense en la EM supera la docena de ofertas educativas (con recursos y estructuras curriculares diferentes) y definidas como cita el Compendio de ofertas y servicios del sistema educativo costarricense, "aquella que ofrece un plan de estudio propio con una propuesta programática particular, la cual esté aprobada por Acuerdo del Consejo Superior de Educación y sea permanente en el sistema" (Venegas, 2009, citado por MEP, Dirección de Planificación Institucional, 2017, p. 10). No obstante, todas son sometidas al mismo modelo evaluativo. Por su parte, el segundo factor delimita las posibilidades sociales desde la perspectiva territorial, esto es, las condiciones de desarrollo que las personas de un determinado espacio comparten y el derecho humano a que su acceso sea equitativo.

\section{Presupuestos metodológicos}

La investigación siguió la ruta metodológica propia de un estudio de carácter diagnóstico, que abarcó diferentes niveles de análisis: exploratorio, descriptivo y correlacional, en función del grado de profundidad de los objetivos planteados. No obstante, para los fines del presente artículo, solo se muestran análisis de los dos primeros. De manera tal que la investigación desarrolló una estrategia mixta, que facilitó la validación de los hallazgos, mediante el empleo de métodos cuantitativos y cualitativos en la interpretación de los datos con el propósito de fortalecer la complementariedad de ambos enfoques (Hernández, Fernández y Baptista, 2010).

En el plano cualitativo, se realizaron 22 entrevistas semiestructuradas en profundidad, utilizando guías para su ejecución y diseñadas en función de las particularidades de los diferentes sujetos informantes. Estas incluyeron a exjerarcas del MEP, personal directivo tanto de Direcciones Regionales de Educación (DRE) como de colegios públicos, privados y subvencionados urbanos y rurales; especialistas en evaluación y medición educativa, así como representantes gremiales de educación. También, se aplicaron entrevistas a docentes de las asignaturas sujetas de evaluación y a estudiantes que habían realizado las pruebas. Todas se ejecutaron en el domicilio, lugar de trabajo o de estudio de las personas entrevistadas, con una duración promedio de 1 hora y luego de la debida firma del consentimiento informado. El componente cuantitativo, particular del objetivo 3, abarcó análisis estadísticos a partir del diseño de bases propias, elaboradas según información proporcionada por la DGEC y del

Francisco González-Alvarado, Ignacio Acosta-Ballestero, Cindy Vanessa Artavia-Aguilar, Guillermo Calderón-Ramírez, Pablo Chaverri-Chaves, Eddy Cruz-Arroyo, Andrea Ramírez-González, Magaly Rodríguez-Pineda y José Solano-Alpizar

Los artículos de la Revista Electrónica Educare del Centro de Investigación y Docencia en Educación de la Universidad Nacional, Costa Rica, se comparten bajo términos de la Licencia Creative Commons: Reconocimiento, № Comercial, Sin Obra Derivada 3.0 Costa Rica. Las autorizaciones adicionales a las aquí delimitadas se pueden obtener en el correo: educare@una.cr 
Departamento de Estadística del MEP. Estas incluyeron resultados de las pruebas (cohorte del 2016); registros de carácter sociodemográficos; socioeducativos e información particular del índice de desarrollo social 2017 (IDS) proporcionado por el Ministerio de Planificación Nacional y Política Económica (MIDEPLAN). Todos los datos fueron asociados a la población estudiantil examinada y a los centros educativos donde realizaron las evaluaciones.

Del total de 40.541 estudiantes que aplicaron las PNB-EM en el 2016, se obtuvieron 37.356 casos (población de estudio), en los que se logró asociar, mediante la construcción de una base de datos inédita, el distrito electoral con el correspondiente distrito administrativo de residencia, para una diferencia de 3.185 casos en los que este registro no pudo ser asignado, debido a falta de información con respecto al distrito electoral. A partir de ello, se estableció la ubicación de la población estudiantil entre las distintas categorías de clasificación propias del IDS-2017: áreas de mayor y menor desarrollo relativo (AMMDR) de manera que se pudo establecer referencias respecto a las condiciones sociales de los lugares de residencia de la población estudiantil. El análisis de los resultados de las pruebas de bachillerato consideró la aplicación de la denominada "curva","criterio técnico- político"empleado por la DGEC con la autorización del ministro o ministra del momento, que reconoce puntos adicionales (8 puntos) a la calificación final de la PNB-EM para cada asignatura. De igual forma se utilizó la técnica estadística de clúster para analizar el rendimiento, según las diferentes modalidades educativas de la educación media.

\section{Presentación y discusión de resultados}

\section{Los objetivos de las PNB-EM en perspectiva histórica}

Como se menciona en el resumen general del informe de investigación Balance critico de objetivos, modelo evaluativo, resultados y utilidad de la información las Pruebas Nacionales de Bachillerato en Educación Media como instrumento de certificación del conocimiento de estudiantes, las PNB-EM"se restablecieron con fines de certificación de los aprendizajes en el año de 1988 en el marco de un contexto histórico educativo de América Latina que experimentó un largo y complejo proceso de modernización del Estado caracterizado, entre otros aspectos, por la adopción de una serie de reformas orientadas a la estabilización macroeconómica de sus países" (González et al., 2018, p. 12). En correspondencia con estos procesos de reforma del Estado, los diferentes gobiernos iniciaron una profunda discusión acerca del tema de la calidad de la educación y de las políticas educativas que se debían aprobar para alcanzarla. Es así como surgen una serie de cambios en los sistemas educativos orientados a la consecución de una educación de calidad, las cuales armonizaban con el enfoque ideológico hegemónico en la región, en el que las teorías sobre el capital humano daban sustento a una visión de la educación y del conocimiento como ejes de la transformación productiva en América Latina (Braslavsky, 1999; Solano, 2001; UN. CEPAL Y UNESCO. OREALC, 1992). Al respecto, el exministro de Educación Francisco A. Pacheco, quien en su gestión (1986-1990) restableció las PNB-EM, acotó:

Francisco González-Alvarado, Ignacio Acosta-Ballestero, Cindy Vanessa Artavia-Aguilar, Guillermo Calderón-Ramírez, Pablo Chaverri-Chaves, Eddy Cruz-Arroyo, Andrea Ramírez-González, Magaly Rodríguez-Pineda y José Solano-Alpizar 
doi: http://doi.org/10.15359/ree.24-1.13

URL: http://www.una.ac.cr/educare

CORREO: educare@una.cr

En efecto, la intención nuestra es que estas pruebas se sigan administrando en tercero y sexto grados y en tercero y quinto años. Se trata de los años en que concluyen los ciclos de nuestra educacion formal y los exámenes han de tener por ello un carácter comprensivo de los conocimientos adquiridos en ese periodo. Si queremos asegurar los efectos beneficiosos que estas primeras experiencias le han traído a la educaci6n nacional, debemos incorporarlas al sistema regular de promoción y convertirlas en verdaderos exámenes, cosa que ocurrirá, según lo esperamos, a finales de 1988. (MEP, 1986-1990, p. 35)

Como bien lo deja anotado el exministro, la calidad se convirtió en el eje central de las reformas educativas. En el marco del contexto histórico-educativo regional señalado, el país toma nuevamente la decisión de certificar los aprendizajes de la población estudiantil que concluye la Educación Media, esta vez mediante la realización de pruebas de carácter nacional y de tipo estandarizada (los exámenes de bachillerato se aplicaron en forma continua entre 1954 y 1973). Entre las principales razones que esgrimió el exministro Pacheco está "un estancamiento general en el desarrollo intelectual de los jóvenes" (MEP, 1986-1990 citando a Méndez, Chaves y Escalante, 1981, p. 31); "niveles de eficiencia [del sistema educativo] que antes parecían satisfactorios, hoy nos resultan a muchos, inadmisibles ... la anarquía que ya habíamos podido apreciar en lo que se refiere al cumplimiento de los programas vigentes" (MEP, 1986-1990, pp. 31-32).

Observaciones que conducen en primera instancia a la aplicación en 1986 y 1987 de pruebas de carácter diagnóstico en las materias de Español y Matemáticas, para los niveles de sexto, noveno y undécimo años. Las apreciaciones descritas, unidas a los denominados "efectos" de los resultados de esas pruebas, contribuyeron decisivamente a que se transformaran de un carácter diagnóstico a otro de certificación. El máximo jerarca de educación, Francisco Antonio Pacheco, en su momento argumentó entre los efectos que constituyen medios de control para los maestros, las maestras, los directores, las directoras y el MEP están promover un sano orgullo institucional; se ha despertado el interés de los padres y las madres de familia por la educación de sus hijos e hijas; los maestros y las maestras reconocen que deben de realizar mayores esfuerzos (MEP, 1986-1990).

En las principales observaciones referidas a los objetivos que sustentaron las PNB-EN resaltan las siguientes:

- Tonando como referencia las valoraciones de los sujetos entrevistados, prevalecía un conocimiento parcial y fragmentado sobre estos. A la vez coinciden en la importancia de revisarlos a profundidad, fundamentalmente porque constituyeron más un conjunto de aspiraciones sobre el sistema educativo nacional como un todo, que una orientación puntual y precisa sobre la realización de las pruebas.

- No respondían a las expectativas que pretendían orientar, especialmente en cuanto a la promoción de la calidad de la educación y a la viabilidad de su aplicación a todas las modalidades de la EM y los correspondientes contextos sociales.

Francisco González-Alvarado, Ignacio Acosta-Ballestero, Cindy Vanessa Artavia-Aguilar, Guillermo Calderón-Ramírez, Pablo Chaverri-Chaves, Eddy Cruz-Arroyo, Andrea Ramírez-González, Magaly Rodríguez-Pineda y José Solano-Alpizar 
- Los objetivos que orientaron la realización de las PBN-EM redactados 30 años atrás, no experimentaron prácticamente modificaciones desde su formulación.

\section{Caracterización general de los criterios técnicos y metodológicos del modelo evaluativo}

De acuerdo con el documento Informe Nacional. Pruebas Nacionales de Bachillerato de la Educación Formal del año 2016, elaborado por la DGEC, las PNB-EM desde su restablecimiento se estructuraron con base en los modelos de evaluación educativos referidos a normas (19881994,1999-2018) y a criterios (1995-1998), este último denominado en el contexto nacional "modelo por objetivos", con el cual se pretendió hacer una asociación de los modelos citados; no obstante, su puesta en ejecución fue muy corta y se optó por el primero. Este modelo se fundamenta en el paradigma psicométrico en el que, de acuerdo con Gronlund y Linn (1990), la medición del desempeño es interpretable en términos de posiciones relativas entre los individuos y entidades. Desde esta premisa, la DGEC justifica su aplicación al ámbito de las PNBEM, porque "al modelo de normas se le atribuyen fortalezas como la interpretación objetiva de los resultados, la comparación del individuo con los del grupo y entre grupos y la utilidad para seleccionar individuos (acreditación, admisión, becas, aprovechamiento escolar, entre otras" (MEP, 2016, p. 9).

Dentro del marco de instrumentos normativos sobre las PNB-EM, el Reglamento de Evaluación de los Aprendizajes (REA) contempló un capítulo exclusivo para tutelarlas y la DGEC se encargó del proceso completo de diseño, evaluación y elaboración de informes. Los exámenes se fundamentaron en los objetivos y los contenidos de los programas de estudio de 6 asignaturas del denominado ciclo de la Educación Diversificada (dos últimos años de la Educación Media): Matemáticas, Español, Estudios Sociales, Educación Cívica, una ciencia: Biología, Química o Física y un idioma extranjero: Inglés o Francés. La calificación final de las PNB-EM para cada estudiante postulante en las materias objeto de medición se determinó por la combinación porcentual de dos componentes: la calificación obtenida en el examen de bachillerato (EB) de la respectiva prueba con un valor de un $60 \%$ y un rubro denominado Nota de Presentación (NP) que incluye el promedio de los últimos 5 periodos lectivos del ciclo de la Educación Diversificada, con un valor de 40\%; criterio que se empleó desde su restablecimiento y que procuró reconocer la importancia de ponderar los aprendizajes adquiridos durante los dos últimos años de la EM, no obstante, la distribución porcentual adoptada inclinó el peso mayor y prácticamente definitorio a los resultados del EB.

Principales observaciones al modelo referido a normas:

- El empleo de evaluaciones estandarizadas en el contexto del modelo referido a normas, contribuyó a que sus resultados pudiesen catalogarse como de altas consecuencias, tal y como se expondrá en detalle en el siguiente apartado. 
doi: http://doi.org/10.15359/ree.24-1.13

URL: http://www.una.ac.cr/educare

CORREO: educare@una.cr

- Con la aplicación del modelo, la interpretación de los resultados de las pruebas se distingue por presentar información sobre el desempeño promedio institucional y regional, así como, del desempeño de los examinados con respecto a la dificultad estimada de los ítems particulares de cada asignatura. Datos que resultan pertinentes para tomar decisiones relativas a la construcción técnica de la prueba. Sin embargo, no aportan información significativa para el desarrollo de estrategias pedagógicas de trabajo entre docentes.

- Privilegia la evaluación de las asignaturas denominadas básicas en detrimento de otras que también integran el currículum de la EM, esencialmente las artes y las humanidades.

- Se distingue por su calidad técnica: confiabilidad y validez (enfocada a la validez del constructo del ítem), así como por lo sistemático de la información sobre el desempeño que genera al finalizar de cada aplicación anual. No obstante, deja de lado aspectos de validez cultural (sensibilidad cultural), tanto en la formulación como en la ponderación de los resultados.

\section{Perfil exploratorio de la población estudiantil y la oferta de educación media según el rendimiento obtenido en las PNB-EM y el IDS distrital}

\section{Perfil sociodemográfico}

El perfil sociodemográfico resalta por las siguientes características: la mayor cantidad de estudiantes tiene edades menores de 18 años; son más el número de mujeres; residen en la Gran Área Metropolitana (GAM), mayoritariamente en San José, la capital del país, y provienen de los distritos ubicados en el nivel de desarrollo medio según el IDS-2017. A continuación, se profundiza en los rasgos de perfil.

- La estructura por sexo de la población estudiada revela que el grupo mayoritario de la población estudiantil que realizó las pruebas fueron mujeres, quienes representan aproximadamente un $56 \%$ del total de estudiantes.

- El mayor grupo de estudiantes que realizó las pruebas está representado por jóvenes de 18 y menos años con el $63.94 \%$; el $36 \%$ restante las hicieron con una edad superior a la mayoría de edad. Esta última característica pone en evidencia que prácticamente 3 de cada 10 estudiantes realizan las pruebas en condición de rezago.

- De acuerdo con la clasificación de las AMMDR según el IDS distrital del 2017, la mayoría de la población estudiantil examinada vivía en un distrito con IDS medio, los cuales agrupan prácticamente al $50 \%$ del estudiantado. En segundo lugar, los que pertenecen a distritos con IDS bajo o muy bajo que concentran al $30,25 \%$ de la población estudiantil y en menor medida los que residen en distritos con IDS alto quienes representan el $23,16 \%$. 
- A nivel provincial, prácticamente, 1 de cada 3 estudiantes residían en San José (31\%) la capital del país, seguidos por Alajuela con un $20 \%$. El resto de la población estudiantil, es decir cerca del 50\%, habitaban en las provincias de Cartago, Heredia, Puntarenas, Limón y Guanacaste, registrando cada una de estas en porcentajes en un rango del $8 \%$ al $11 \%$ aproximadamente (Ver Figura 1 ).

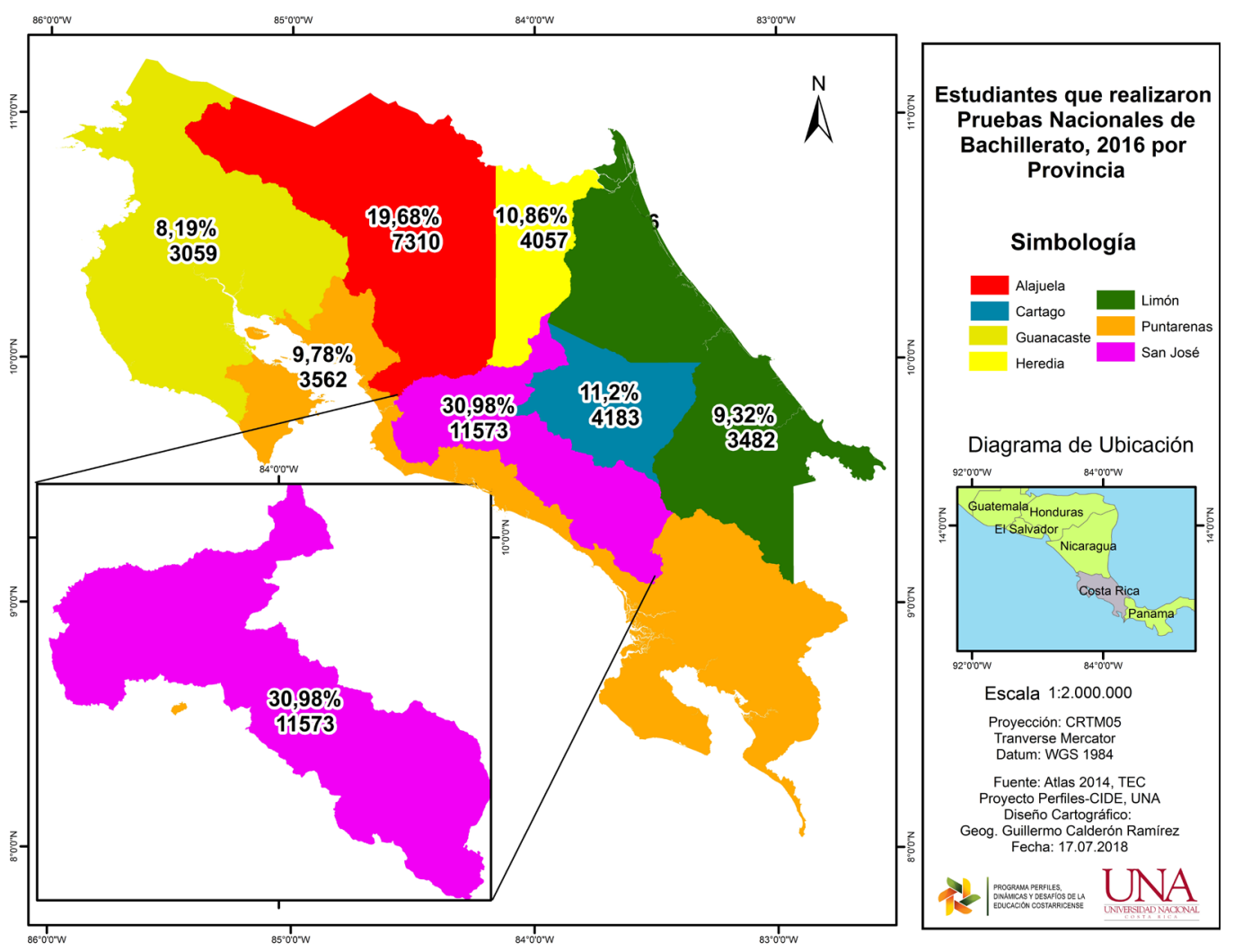

Figura 1. Mapa de estudiantes que realizaron las PNB-EM 2016 por provincia.

Nota: Elaboración propia.

\section{Perfil socioeducativo}

El perfil socioeducativo resalta por las siguientes características: en el caso de quienes obtuvieron mejores rendimientos (mayores niveles de aprobación) son, en su mayoría, hombres, menores de 18 años, residen en la GAM, mayoritariamente en la capital, pertenecen a las Direcciones Regionales de Educación (DRE) ubicadas en también en el centro del territorio nacional y provienen de los distritos ubicados en las AMMDR de niveles medio y alto. No requieren, en su gran mayoría, de la curva para aprobar las evaluaciones y sus notas de presentación están por encima del promedio general, al igual que las correspondientes al promedio de los exámenes. 
doi: http://doi.org/10.15359/ree.24-1.13

URL: http://www.una.ac.cr/educare

CORREO: educare@una.cr

En el caso de la población estudiantil que obtuvo los rendimientos más bajos (mayores niveles de aplazamiento), son en su mayoría mujeres, mayores de 18 años, residen fuera de la GAM, mayoritariamente en las DRE ubicadas en las áreas fronterizas y costeras; y provienen de los distritos ubicados en las AMMDR de niveles bajo y muy bajo. Requieren en su gran mayoría de la curva para aprobar las PNB, sus notas de presentación están ligeramente por debajo del promedio general y el promedio de los exámenes es aproximadamente 10 puntos menos que aquellos que viven en las AMMDR de nivel alto. A continuación, se profundiza en los rasgos de perfil.

- El promedio de aprobación nacional fue de $73,48 \%$; en consecuencia, el $26.52 \%$ de la población estudiantil aplazaron en 1 o más de las 6 asignaturas sujetas de medición. No obstante, al analizar los resultados con o sin la aplicación de la curva, solamente un $41,15 \%$ lo hacen sin ayuda de este criterio "técnico-político" (aproximadamente 4 de cada 10 que realizan las pruebas) y el 32,33\% solo lo logra al aplicarse esta (Véase Tabla 1). Situación que pone en evidencia que los resultados obtenidos por la población estudiantil resultan altamente dependientes de la aplicación de la "curva".

Tabla 1: Total de estudiantado aprobado y aplazado con curva y sin esta, 2016

\begin{tabular}{llll}
\hline & Aplazado & Aprobado & Total \\
\hline Sin curva & $67,67 \%$ & $32,33 \%$ & $100 \%$ \\
Con curva & 26,52 & 73,48 & $100 \%$ \\
\hline
\end{tabular}

Nota: Elaboración propia.

- De la población de estudiantes que comprende el estudio, un total de $31470(84,24 \%)$ asisten a colegios públicos, 4697 a colegios privados $(12,57 \%)$ y $1189(3,18 \%)$ a colegios subvencionados. La condición de aplazado y aprobado, de acuerdo con esta característica, refleja que la población estudiantil de colegios subvencionados ostenta un porcentaje mayor de aprobados $(86,96 \%)$, seguido de los colegios privados $(79,28 \%)$ y, por último, los colegios públicos con un porcentaje significativamente menor (33,73\%). La aplicación del criterio de la curva modifica estas proporciones, porque duplica el porcentaje de población aprobada proveniente de colegios públicos $(69,41 \%)$. En el caso de los colegios subvencionados y privados, una vez aplicada la curva, la cifra de aprobados pasa como mínimo al 94\% (Véase Figura 2). 


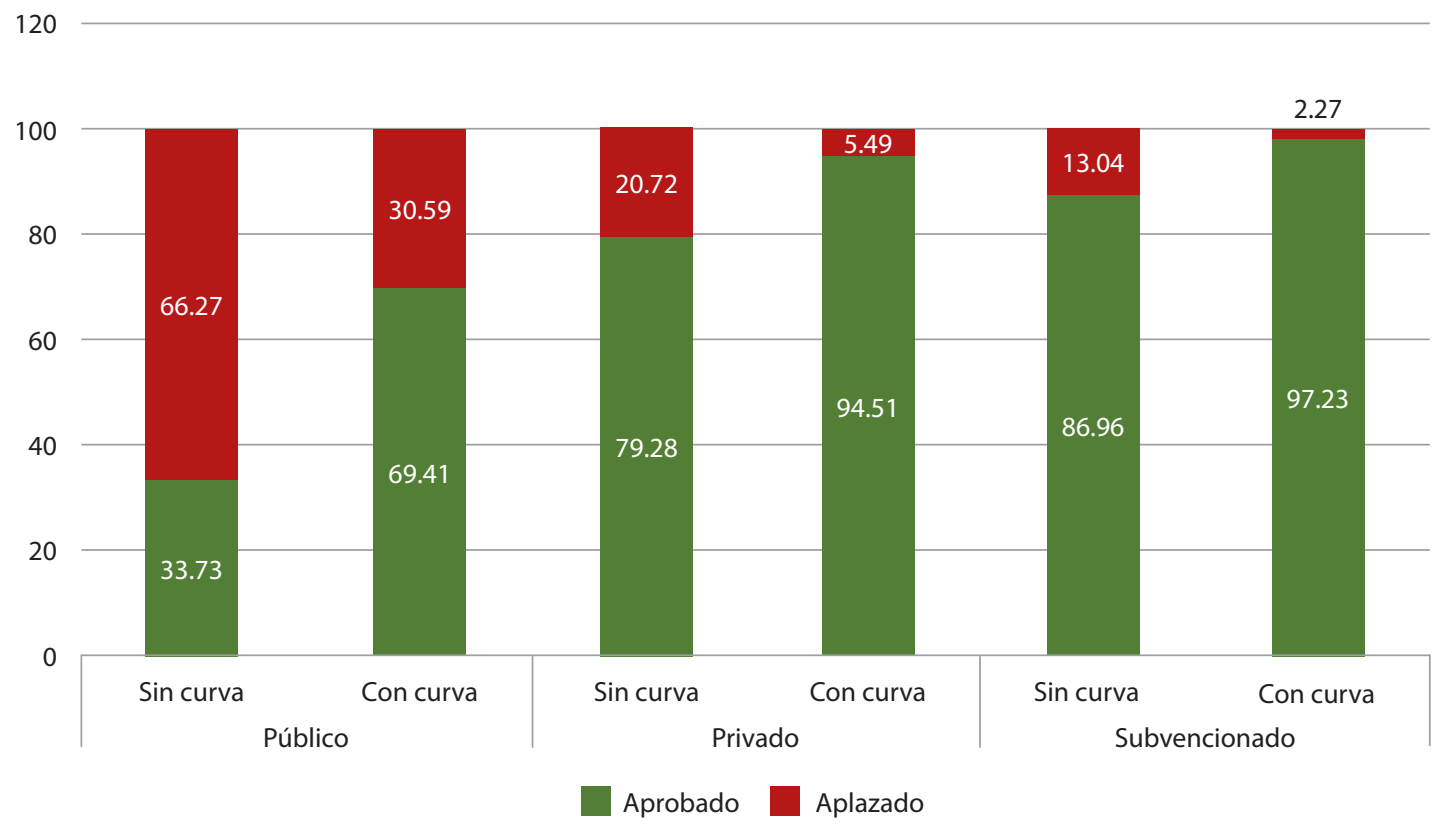

Figura 2. Porcentaje de estudiantes aprobados o aplazados según tipo de financiamiento del colegio, sin curva y con curva.

Nota: Elaboración propia.

- Según los datos analizados, se puede apreciar una leve tendencia a que los hombres muestran un mayor rendimiento que las mujeres. Visto por asignatura, en matemática se presenta el menor rendimiento, tanto en hombres como en mujeres, contrario a inglés, que corresponde a la asignatura baja para ambos sexos, ya que solo el $38,69 \%$ de las mujeres consigue aprobar todas las asignaturas y el $44,26 \%$ de los hombres lo logra. Al aplicarse la curva, los cambios son sustantivos, pues las mujeres pasan a aprobar en un $71,55 \%$, mientras que los hombres aprueban en tres de cada cuatro casos $(75,9 \%)$, con mayor rendimiento para ambos sexos.

- Quienes aplicaron las pruebas con 17 años o menos las aprobaron en el $84,48 \%$ de los casos, mientras que el grupo de estudiantes con 21 o más años al momento de hacerlas, las aprobaron en solamente el $55 \%$ de los casos, lo cual representa casi 30 puntos porcentuales de diferencia entre estos grupos de edad. Estos resultados son con la curva aplicada, pues sin la misma las diferencias serían aún mayores. De esta manera, sin la curva, solo un 17,9\% de quienes tienen 21 o más años aprobaron, mientras que el $55,7 \%$ de estudiantes de 17 años o menos lo logró. Lo anterior implica que sin la aplicación de la curva se presenta una diferencia del $37,9 \%$ en el nivel de promoción entre estos dos grupos etarios. 
doi: http://doi.org/10.15359/ree.24-1.13

URL: http://www.una.ac.cr/educare

CORREO: educare@una.cr

- Las personas de zona urbana obtuvieron un promedio de nota de examen de 72,06 y las personas de zona rural uno de 67,36, que representa una diferencia de casi cinco puntos $(4,7)$. En la zona rural, sin la aplicación de la curva, casi uno de cada tres estudiantes aprobó las pruebas (32,75\%), mientras que, con la aplicación de la curva, la aprobación sube a más de dos tercios (67,17\%). Es decir, que el efecto de la curva en la zona rural incide en mayor medida a una tercera parte de la población evaluada $(34,42 \%)$. En la zona urbana, se tiene que la aprobación sin curva fue del $44,6 \%$, pero con la aplicación de la curva aumenta a $76,05 \%$, es decir, un aumento de $31,5 \%$ que apenas representa 3 puntos porcentuales menor al efecto de la zona rural. Entonces, mientras que menos de la mitad de los examinados aprobaron sin la curva en la zona urbana, esta zona aumenta el nivel de aprobación de los examinados a más de tres cuartas partes cuando se implementa la curva.

- Las personas que viven en distritos ubicados en AMMDR de nivel alto, presentan un nivel de aprobación (sin curva) del 55,62\% y aquellas que se encuentran en AMMDR de nivel muy bajo aprueban (también sin curva) en apenas el 25,08\% de los casos. En otras palabras, mientras más de la mitad de quienes tienen un IDS alto en su distrito de residencia aprueba los exámenes de bachillerato sin que se aplique la curva, solamente 1 de cada 4 estudiantes de muy bajo IDS lo consigue (sin la curva). Cuando se aplica la curva, estas grandes diferencias identificadas tienden a reducirse, pues el porcentaje de aprobado de las AMMDR de nivel muy bajo, sube a casi un $60 \%$, equivalente a un aumento de cerca del $35 \%$. En otras palabras, la diferencia entre antes y después de aplicada la curva influye en la aprobación de más de 1 de cada 3 estudiantes de las AMMDR de nivel muy bajo. En cuanto a las personas que viven en distritos con IDS alto, aunque su realidad es bastante diferente, la curva no deja de tener un efecto amplio. En esta condición, la aprobación pasa del $55,6 \%$ sin curva, al $82,8 \%$ con la aplicación de la curva, lo cual muestra una diferencia del $27,2 \%$ entre ambos escenarios. Por lo tanto, la aplicación de la curva beneficia a 1 de cada 4 estudiantes (Véase Figura 3).

- Otro análisis de interés consiste en ver la relación entre los diferentes niveles de las AMMDR del distrito de residencia de la población estudiantil, según el efecto de la curva en relación con el número de asignaturas que se aprueban. Al respecto, se determinó que las personas que habitan en las AMMDR con niveles bajos y muy bajos se ven más beneficiadas, pues con la aplicación de la curva aprueban 1,5 asignaturas más que aquella población estudiantil proveniente de las AMMDR de nivel alto, quienes se ven beneficiados en 0,73 materias. Si se clasifica el rendimiento entre los niveles de las AMMDR, se puede observar una tendencia diferenciada según el nivel particular del distrito en que se vive. Así, mientras que de los 1450 estudiantes que obtienen una calificación de excelente en las PNB, el 90\% residen en distritos con IDS nivel alto y medio (1315 estudiantes), solo el 10\% de los que viven en los distritos con IDS bajo y muy bajo lo alcanzan (135 estudiantes). 


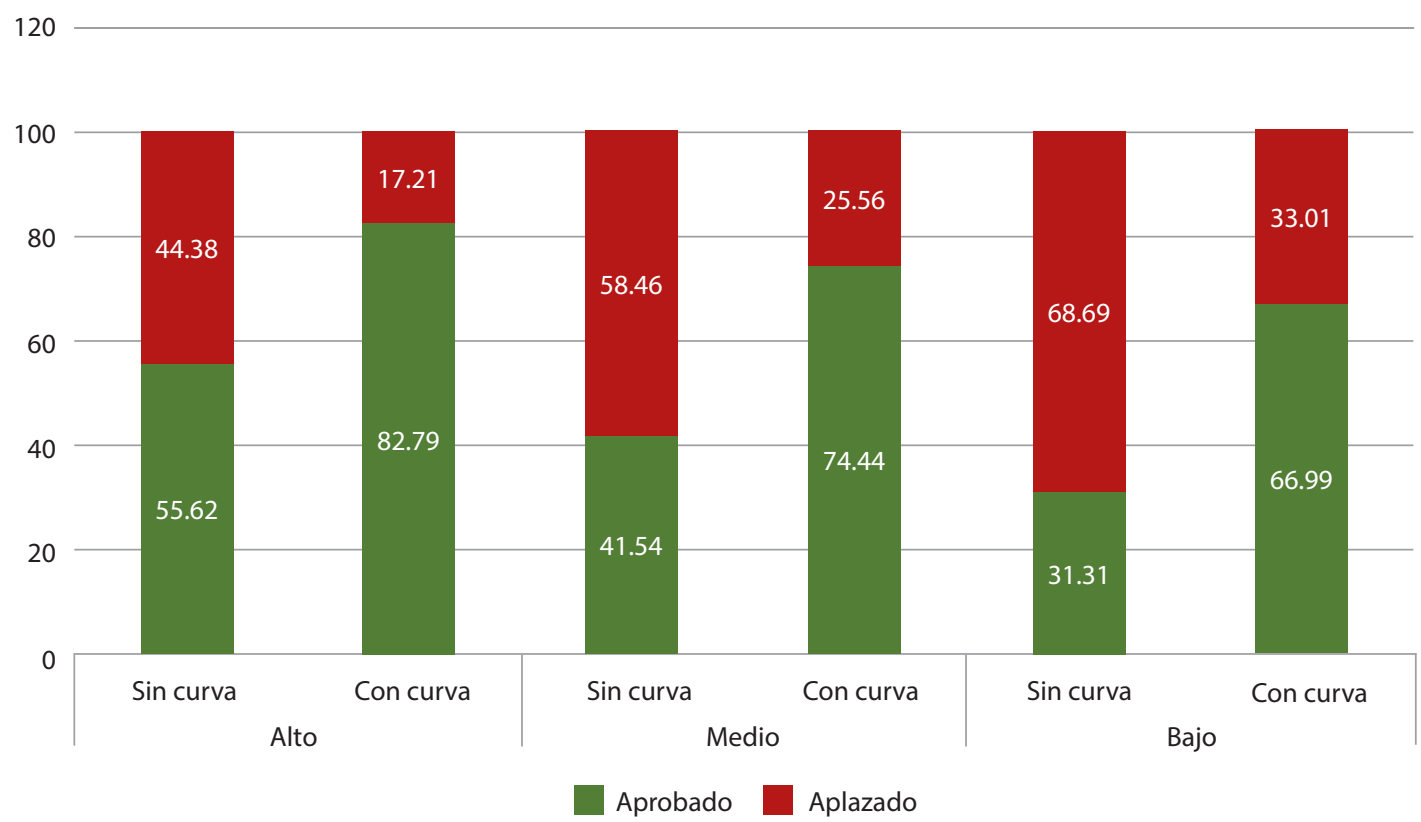

Figura 3. Porcentaje de estudiantes aprobados o aplazados según IDS, sin curva y con curva. Nota: Elaboración propia.

- Si se analiza el rendimiento al interior de cada uno de los niveles de categorización del IDS distrital, las diferencias en cuanto a las calificaciones son igualmente significativas. Mientras que solo el 30\%, 2680 del total de la población estudiantil procede de distritos con IDS de nivel alto se ubica en el rango de notas deficientes, es decir, que aplazan las PNB-EM al menos en 1 asignatura, en el caso de estudiantes de los distritos de nivel muy bajo, representan el $70 \%$. Ello evidencia que la procedencia territorial de la población estudiantil y las condiciones de desarrollo de esos lugares reflejan rendimientos desiguales en las pruebas nacionales.

- El promedio de las notas de presentación y el rendimiento promedio en los exámenes de cada asignatura, según los niveles de las AMMDR, también muestran un comportamiento escalonado. De esta manera, conforme más alto son estas, más alto tiende a ser no solo el nivel de aprobación (con curva o sin curva, como ya se indicó), sino también la nota promedio de examen. De este modo, las personas que residen en las AMMDR de nivel alto obtuvieron una calificación media de 75,37 mientras que las personas de las AMMDR de nivel muy bajo presentaron una nota de 64,3; es decir, 11 puntos por debajo de las personas ubicadas en niveles altos de las AMMDR. 
doi: http://doi.org/10.15359/ree.24-1.13

URL: http://www.una.ac.cr/educare

CORREO: educare@una.cr

- El MEP tiene una estructura regional de 27 DRE que abarcan todo el territorio nacional. Así, mientras las DRE ubicadas en la GAM presentan los mejores rendimientos, las regiones periféricas, fronterizas y costeras muestran los rendimientos más bajos. En este sentido, mientras la DRE de Sulá en Talamanca muestra el nivel más bajo de aprobación sin curva, pues apenas el $15,5 \%$ de sus estudiantes aprobaron el bachillerato sin la aplicación de este criterio, la región educativa de Cartago obtuvo un 52,4\% de nivel de promoción también sin la curva, lo que representa una diferencia en proporción de un $36,9 \%$, lo cual implica una brecha muy grande de rendimiento.

\section{Perfil de las ofertas educativas}

El rendimiento obtenido en las PBN-EM ha sido caracterizado por diferentes estudios, tal y como se mencionó, en función exclusiva de los resultados obtenidos de acuerdo al tipo de financiamiento del centro educativo: privados, subvencionados y públicos, con la limitación de que el enfoque adoptado no permite analizar las diferencias prevalentes al interior de las variadas ofertas educativas que distingue a la educación media pública, a pesar de la variedad que las caracteriza. En este sentido, se logró incluir en el estudio información particular de las ofertas de EM mediante la realización de una tipología de las modalidades según el rendimiento obtenido, con base en la simetría que se presenta en la distribución y un análisis de clúster, que:

es una técnica de clasificación que sirve para poder detectar y describir subgrupos de sujetos o variables homogéneas en función de los valores observados dentro de un conjunto aparentemente heterogéneo. Se [basa] en el estudio de las distancias entre ellos, [lo cual permite] en el análisis, cuantificar el grado de similitud, en el caso de las proximidades, y el grado de diferencia, en el caso de las distancias. Como resultado aparecen agrupaciones o clúster [cursivas añadidas] homogéneos. (Vilà, Rubio-Hurtado, Berlanga-Silvente y Torrado-Fonseca, 2014, p. 114)

Para lo anterior, se usó como variable cuantitativa el promedio de bachillerato que incluye la nota de colegio y nota de exámenes, cuyo ajuste resultó ser de 0,6-el cual se considera bueno, es decir, los casos se encuentran en el centro de su conglomerado- y se logró reconocer tres estratos: los que se sitúan en la cola izquierda de la curva, que agrupó a los colegios con mayor rendimiento, los que se encuentran en el centro de la distribución, que incluyó a los colegios con resultados dentro del promedio y a los que se hallan en la cola derecha, que según reveló los colegios con menores rendimientos. Resultando la distinción de un perfil exploratorio de 3 estratos, con los siguientes resultados:

- Estrato 1 compuesto por colegios académicos privados, subvencionados, científicos, humanísticos y experimentales bilingües: obtiene los niveles de aprobación y rendimiento por encima de la media general; agrupa mayoritariamente mujeres; con 
doi: http://doi.org/10.15359/ree.24-1.13

promedio de edad de 18,63 años; provienen de distritos cuyo índice de desarrollo social (IDS) es alto y residen en zona urbana, viven principalmente en la provincia de San José y se caracterizan por habitar en la GAM.

- Estrato 2 integra a otros colegios académicos públicos y técnicos profesionales: obtienen los niveles de aprobación y rendimiento en la media general; congrega son mayoritariamente mujeres; con promedio de edad de 18,74 años; proviene de distritos cuyo índice de desarrollo social es medio y residen en zona urbana, viven principalmente en las provincias de San José y Puntarenas y se caracterizan por habitar en la GAM.

- Estrato 3 comprende a los liceos o colegios nocturnos, rurales, telesecundarias, colegios indígenas y los denominadas modalidades "no tradicionales" (IPECS, CINDEA, CONED, IEGB): obtienen los niveles de aprobación y rendimiento por debajo de la media general; integra mayoritariamente mujeres; con promedio de edad de 23,17 años; provienen de distritos cuyo IDS es bajo o muy bajo y residen en zona urbana o rural en casi la misma proporción, viven principalmente en provincias diferentes a San José y se caracterizan por habitar fuera de la GAM.

\section{Resultados del rendimiento según estratos}

La siguiente Tabla 2 permite identificar diferencias significativas respecto al promedio de la nota de presentación y al promedio del rendimiento de las notas de los exámenes entre los estratos con curva y sin curva.

Tabla 2: Promedios de rendimiento en bachillerato con curva y sin curva

\begin{tabular}{lcccc}
\hline Estrato & $\begin{array}{c}\text { Promedio de nota } \\
\text { de presentación }\end{array}$ & $\begin{array}{c}\text { Promedio de } \\
\text { rendimiento exámenes }\end{array}$ & $\begin{array}{c}\text { Promedio de rendimiento } \\
\text { bachillerato sin curva }\end{array}$ & $\begin{array}{c}\text { Promedio de rendimiento } \\
\text { bachillerato con curva }\end{array}$ \\
\hline Estrato 1 & 86.31 & 81.08 & 83.17 & 90.76 \\
Estrato 2 & 82.27 & 69.83 & 74.81 & 82.77 \\
Estrato 3 & 81.86 & 63.57 & 70.89 & 79.17 \\
\hline Total & 82.98 & 70.70 & 56.1 & 83.57 \\
\hline
\end{tabular}

Nota: González et al. (2018, p. 47).

De igual forma, en las Figuras 4 y 5 se distingue que, sin la aplicación de la curva, en el Estrato 3 , ocho de cada diez estudiantes se consideran aplazados, a pesar de haber cumplido un rendimiento satisfactorio durante la etapa de secundaria medida por medio de la nota de presentación. En el Estrato 2, este porcentaje es del 63,65\% el que supera la media general del 58,85\% (Ver Tabla 1). En contraste, el Estrato 1 exhibe un porcentaje de 19,93\% de población aplazada. Estos porcentajes 
doi: http://doi.org/10.15359/ree.24-1.13

URL: http://www.una.ac.cr/educare

CORREO: educare@una.cr

disminuyen considerablemente con la aplicación de la curva, en el Estrato 3 a 25,81\%, en el Estrato 2 a 43,16\% y en el Estrato 1 a 4,77\%. El Estrato 3 es el que más se beneficia con la curva, en promedio aprueban 1,61 exámenes adicionales; en segundo lugar, el Estrato 2 con 1,11 y el Estrato 1 es el menos beneficiado 0,31, debido a que tiene un mayor rendimiento.

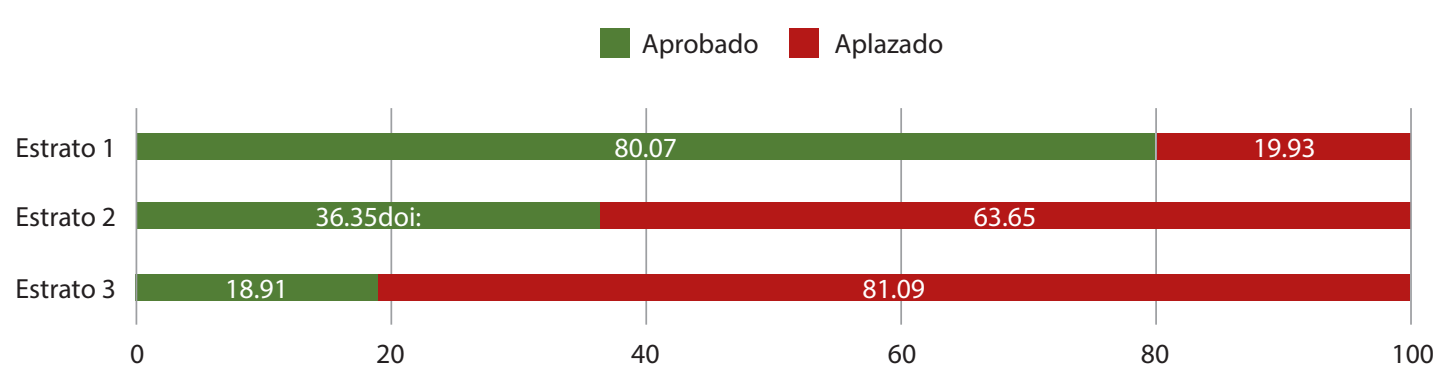

Figura 4. Condición de aprobados y aplazados, estratificados, sin curva.

Nota: Elaboración propia.

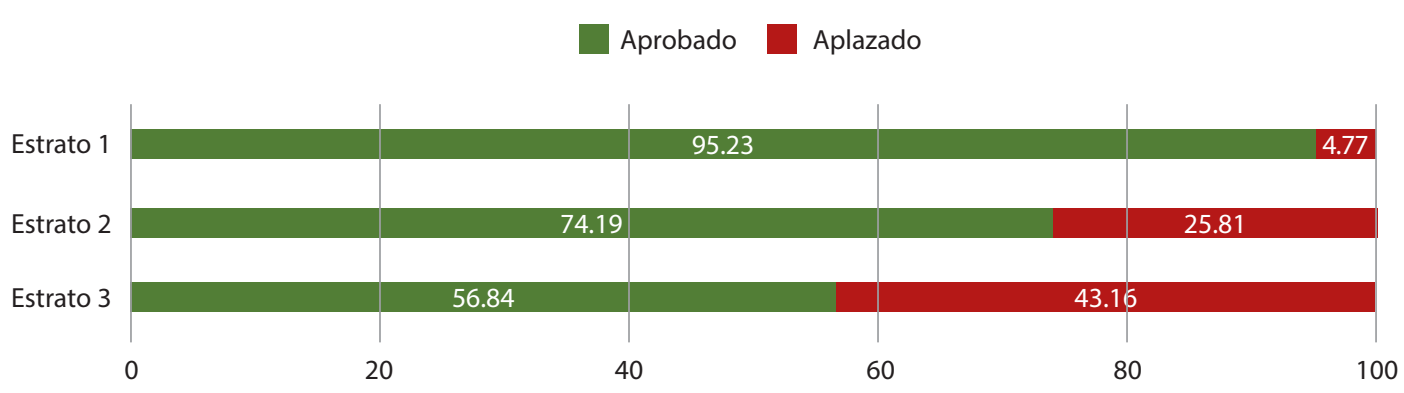

Figura 5. Condición de aprobados y aplazados, estratificados, con curva.

Nota: Elaboración propia.

\section{Valoración general sobre del uso de los resultados de las PNB-EM}

A la DGEC le corresponde la elaboración, administración y calificación de las PNB-EM, que incluye, además, todo un proceso de ensamblaje, revisión de las pruebas, aplicación, calificación, análisis y elaboración de informes de resultados. Estos últimos son comunicados a las DRE anualmente y tienen un carácter nacional. Al respecto se plantean algunas ideas conclusivas:

- La DGEC presenta informes anuales a diferentes instancias del MEP, los cuales presentan gran calidad técnica y permiten el acceso a los resultados de una forma ordenada y sistemática. 
doi: http://doi.org/10.15359/ree.24-1.13

- El uso de los resultados no se vincula directamente con los procesos educativos en el aula y gestión curricular (modificaciones en los programas de estudio).

- No se revela el uso de los resultados en la toma de decisiones relacionadas con la construcción y ejecución de política educativa (gestión del currículo, capacitación docente, orientaciones pedagógicas).

- Los informes técnicos no generan información, orientaciones o conclusiones sobre el uso adecuado de los resultados y las consecuencias de esos usos como parte de un enfoque de validez.

\section{Conclusiones}

El restablecimiento de las PNB-EM con fines de acreditación de los aprendizajes en 1988 respondió a un contexto socioeducativo regional en el cual las concepción de calidad de la educación se asociaba con las nociones de eficacia y eficiencia del sistema educativo. Hoy, tal concepción considera de manera sustantiva la pertinencia y la sensibilidad social como elementos vitales de todo proceso evaluativo y, especialmente, de las políticas educativas nacionales.

Según se expresa en la bibliografía científica consultada, el modelo de pruebas referido a criterios es el más apropiado para la medición de los aprendizajes, lo cual es un argumento compartido por las personas entrevistadas especialistas en evaluación. Sin embargo, un cambio en el modelo de las PNB-EM de normas a criterios, requeriría repensar los propósitos de estas y, sobre todo, las implicaciones que esto traería para el sistema educativo.

El análisis de los resultados de las PNB-EM muestran importantes diferencias en el rendimiento académico según las condiciones socioeconómicas del lugar de procedencia y la oferta educativa. En consecuencia, es muy importante considerar los impactos de estas evaluaciones en función de las condiciones de desarrollo social de los lugares de residencia de los sujetos examinados, así como de las características de la oferta educativa de la que provienen, pues las PBN-EM se diseñan como una "talla única" para personas en condiciones de desarrollo socioeconómico muy desiguales. Este argumento permiten aseverar que estas constituyen pruebas de altas consecuencias para la persona estudiante que las realiza y para la sociedad en su conjunto, especialmente porque la no aprobación impide la certificación de la educación media y limita la posibilidad de continuar con estudios superiores, así como oportunidades de contratación laboral.

Fue posible mostrar una correlación entre las condiciones de desarrollo de las AMMDR según el IDS de los distritos de residencia de la población estudiantil que aplicó las pruebas y el rendimiento obtenido por estos, fue posible caracterizar diferentes perfiles de carácter exploratorio: sociodemográfico, socioeducativo y de modalidades educativas en función del rendimiento obtenido. Las personas de alto rendimiento se caracterizan por vivir en distritos 
doi: http://doi.org/10.15359/ree.24-1.13

URL: http://www.una.ac.cr/educare

CORREO: educare@una.cr

con niveles de desarrollo medios y altos. Mientras que quienes viven en los distritos de nivel bajo y muy bajo obtuvieron los menores puntajes de aprobación y de nota en los exámenes por asignatura, situación que pone en evidencia el alto impacto de las PNB-EM entre la población estudiantil nacional y sugiere un importante nivel de desigualdad en las posibilidades de desempeño de las personas examinadas. Por lo expuesto, se considera que las PNB-EM son reflejo de una sociedad desigual que territorialmente distribuye recursos y oportunidades de manera injusta.

El principio de igualdad de oportunidades que se supone en la realización del examen de bachillerato no se cumple en la realidad, en el tanto el mérito o el logro individual revela importantes vinculaciones con las condiciones socioeconómicas de las AMMDR de los distritos de procedencia de los sujetos examinados y las ofertas educativas existentes, como lo sugieren las correlaciones del rendimiento aquí presentadas con el IDS, así como la estratificación de tipos de centros educativos. De no atenderse las amplias desigualdades aquí mostradas, el sistema educativo se convierte en una inclusión (oferta del sistema educativo) excluyente (examen de bachillerato).

Los resultados de la población estudiantil que realiza las PNB-EM están muy influidos por la aplicación de la "curva" en la nota final. Esta tiene un gran impacto en el aumento de los niveles de aprobación de la mayoría de la población estudiantil, y de manera particular entre aquella que proviene de los distritos con IDS bajo y muy bajo, y de zona rural. Sin la "curva" los porcentajes de aprobación nacional serían realmente calamitosos, situación que hace preocupante la calidad y nivel de desempeño de la oferta educativa media.

Respecto a los informes anuales generados por la DGEC sobre los resultados de las PNB, resalta la calidad técnica de estos mismos; no obstante, prevalecen algunas limitaciones, entre las que resaltan: su escaso aprovechamiento para el mejoramiento de los procesos educativos en el aula y la gestión curricular de las instituciones, su poca articulación con otras instancias del MEP para que el uso de los resultados tenga repercusiones en la toma de decisiones relacionadas con el diseño y ejecución de políticas educativas, la poca orientación acerca de la utilidad de los resultados y las consecuencias de estos como parte de un enfoque de validez integral que contribuya al mejoramiento de los procesos educativos y a la gestión curricular y pedagógica en los centros escolares.

\section{Declaración de Material complementario}

Este artículo tiene disponible como material complementario:

- Laversión preprintdelartículo en https://www.repositorio.una.ac.cr/handle/11056/15202

- Los instrumentos de entrevista mencionados en la Metodología. Para acceder a ellos puede visitar la página del artículo en https://doi.org/10.15359/ree.24-1.13 
doi: http://doi.org/10.15359/ree.24-1.13

URL: http://www.una.ac.cr/educare

CORREO: educare@una.cr

\section{Referencias}

Artavia-Aguilar, C. V., Calderón-Ramírez, G., Ramírez-González, A., González-Alvarado, F., Chaverri-Chaves, P., Cruz-Arroyo, E. (2019). Análisis de los resultados de las pruebas nacionales de bachillerato de la educación media de los colegios que conforman las direcciones regionales de educación de zonas fronterizas costarricenses. 2013-2016. Revista Electrónica Educare, 23(3), 1-23. https://doi.org/10.15359/ree.23-3.1

Braslavsky, C. (1999). Re-haciendo escuelas: Hacia un nuevo paradigma en la educación latinoamericana. Buenos Aires: Santillana.

Chaverri, P., Cruz, E. y González, F. (2018). Percepción de la población costarricense en relación con las pruebas nacionales de bachillerato: Balance comparativo 2007-2017. En M. Castro Pérez (Coord.), Memoria IV Congreso Iberoamericano de Pedagogía: "Sueños y utopías que inspiran transformación" (pp. 199-212). Heredia, Costa Rica: Universidad Nacional, CIDE. https://www.dropbox.com/s/jp8sstlwl6w869f/Memoria\%20CIP\%202018.pdf?dl=0

Consejo Superior de Educación (CSE). (2018). Acta CSE-SG-143-2019. San José, Costa Rica: Ministerio de Educación Pública.

Esquivel, J., Montero, E., Sosa, D, Hernández, A., Corella, M. y Fallas, J. (2006). Evaluación externa de las Pruebas Nacionales de Bachillerato de la Educación Media (Informe final). San Pedro, Costa Rica: Consejo Superior de Educación.

González, F., Artavia, C., Calderón, G., Chaverri, P., Cruz, E., Ramírez, A., Rodríguez, M. y Solano, J. (2018). Balance crítico de objetivos, modelo evaluativo, resultados y utilidad de la información de las Pruebas Nacionales de Bachillerato en Educación Media como instrumento de certificación del conocimiento de estudiantes. Heredia, Costa Rica: UNA, CIDE-INEINA. https://drive.google.com/file/d/1rA1xMf3WheK-cfacKq3xmF8SAUFzmphG/view

Gronlund, N. E. y Linn, R. L. (1990). Measurement and evaluation in teaching (6 ed.). New York: MacMillan.

Hernández, R., Fernández, C. y Baptista, P. (2010). Metodología de la investigación. México: McGraw-Hill.

Ministerio de Educación Pública (MEP). (1986-1990). Memoria final. Periodo 1986-1990. San José, Costa Rica: Autor. http://www.asamblea.go.cr/sd/Memoriasgobierno/Memoria\%20 Ministerio\%20de\%20Educaci\%C3\%B3n\%20P\%C3\%BAblica\%201986-1990-1.pdf

Ministerio de Educación Pública (MEP). (2016). Informe Nacional. Bachillerato de la Educación 
doi: http://doi.org/10.15359/ree.24-1.13

URL: http://www.una.ac.cr/educare

CORREO: educare@una.cr

Formal 2015. Rendimiento y niveles de desempeño. San José, Costa Rica: DGEC. informe nacional de las pruebas de bachillerato 2015.pdf

Ministerio de Educación Pública (MEP). (2017a). Instructivo para el ensamblaje de las pruebas nacionales de certificación. San José, Costa Rica: Autor.

Ministerio de Educación Pública (MEP). (2017b). Política Educativa: La persona: centro del proceso educativo y sujeto transformador de la sociedad. San José, Costa Rica.

Ministerio de Educación Pública, Dirección de Planificación Institucional. (2017). Compendio de ofertas y servicios del sistema educativo costarricense 2016. San José, Costa Rica: Autor. https://docplayer.es/85140820-Compendio-costarricense-de-ofertas-y-servicios-delsistema-educativo.html

Moreira, T. E. (2002). Estudio correlacional entre las pruebas de bachillerato en la educación media y el rendimiento en educación diversificada. Revista Educación, 26(1), 121-133. https://doi.org/10.15517/revedu.v26i1.2885

Programa Estado de la Nación. (2005). Estado de la Educación Costarricense. San José, Costa Rica: Autor. Recuperado de https://estadonacion.or.cr/informes/?current=3

Sánchez-Mendiola, M.y Delgado-Maldonado, L. (2017). Exámenes de alto impacto: Implicaciones educativas. Investigación en Educación Médica, 6(21), 52-62. https://doi.org/10.1016/j. riem.2016.12.001

Solano, J. (2001). Educación y desarrollo en América Latina: Un análisis histórico-conceptual. Heredia, Costa Rica: EUNA.

UN. CEPAL y UNESCO. OREALC. (1992). Educación y conocimiento: Eje de la transformación productiva con equidad. Santiago de Chile: CEPAL.

Vilà, R., Rubio-Hurtado, M.-J., Berlanga-Silvente, V. y Torrado-Fonseca, M. (2014). Cómo aplicar un clúster jeràrquic en SPSS. REIRE Revista d'Innovació i Recerca en Educació, 7(1), 113-127. 10.1344/ reire2014.7.1717// 